\title{
Morphological Evidence for Adhesive Role of Thin -Symmetric Junctions in Undulating Membranes of Lens Nuclear Fiber Cells
}

\author{
M.J. Costello, K.O. Gilliland, and C.D. Freel
}

Department of Cell and Developmental Biology, University of North Carolina at Chapel Hil, Chapel Hill, NC 27599

The ocular lens contains epithelial cells on the anterior surface and fiber cells throughout the interior. The interface between fiber cells displays unique junctions that change with age. Young fiber cells are interconnected with gap junctions (GJs), $16 \mathrm{~nm}$ thick, that promote exchange by diffusion. After differentiation, the older cells in the lens lose their internal organelles and, as recent evidence suggests, develop fusion pores that create a cellular syncytium [1]. With lage pores available for intercellular transport, the primary role of gap junctions may change to one of adhesion. Fiber cells also have extensive junctions containing orthogonal arrays of the water channel aquaporin-0 (AQP0). Two functions of AQP0 junctions have been proposed: water transport to minimize extracellular space and adhesion [2-4]. Consistent with an earlier model [5], pairs of AQP0 arrays have been described forming flat symmetric junctions [3, 4], similar to gap junctions, but only $14 \mathrm{~nm}$ thick. We report here the presence of these thin-symmetric junctions (TSJs) in high amplitude undulating membranes. Whole fresh human donor lens nuclei were prepared for thinsection TEM (FIG. 1). GJs and TSJs were measured directly correcting for tilt and withFourier averaging to smooth the surface stain variations (FIG. 2). Symmetric junctions were consistently observed within undulating membranes (FIG. 1, 3). The thickness was $14 \mathrm{~nm}$ where curved arrays of AQP0 overlapped (FIG. 3A), 12-15\% thinner than GJs. Comparisons can be readily made when a GJ and TSJ occur in the same membrane pair (FIG. 3B). These observations support the proposal that the TSJs help stabilize the extensive undulating membranes characteristic of aged nuclear fiber cells [6].

References

[1] V.I. Shestopalov and S. Bassnett, J. Cell Sci. 113 (2000) 1913.

[2] G. Chandy et al., J. Membr. Biol. 159 (1997) 29.

[3] M.J. Costello et al., Invest. Ophthalmol. Vis. Sci. 30 (1989) 975.

[4] D. Fotiadis et al., J. Mol. Biol. 300 (2000) 779.

[5] M.J. Costello et al., Proc. 51 $1^{\text {st }}$ Ann. Mtg. EMSA, Cincinnati (1993) 200.

[6] V.L. Taylor et al., Invest. Ophthalmol. Vis. Sci. 37 (1996) 1396.

[7] Supported in part by NIH Grants EY08148 and EY05722.

FIG. 1. Human lens dissection and preparation for thin-section TEM. Upper: Vibratome sections, $200 \mu \mathrm{m}$ thick, are bisected and a mesa (m) is raised to give cross sections of the fiber cells in the embryonic nucleus (en). Other nuclear regions are fetal (fn), juvenile (jn), and adult (an) [6]. Lower: $70 \mathrm{~nm}$ thin sections cut with a diamond knife are collected on TEM grids from which low and high magnification images can be made of the undulating membranes.

FIG. 2. TEM images of junctions are measured directly $(A, D)$ or with Fourier transforms $(B, E)$ reconstructed with filtering to reduce noise (C,F). GJs (A-C) are distinguished from TSJs (D-F). FIG. 3. TEM of undulating membranes. (A) Undulations contain AQP0 arrays (white arrows). Two arrays overlap occasionally to form TSJs (black arrows). (B) GJs between undulations (arrowheads) sometimes appear in the same field as TSJs within undulations (arrows). 

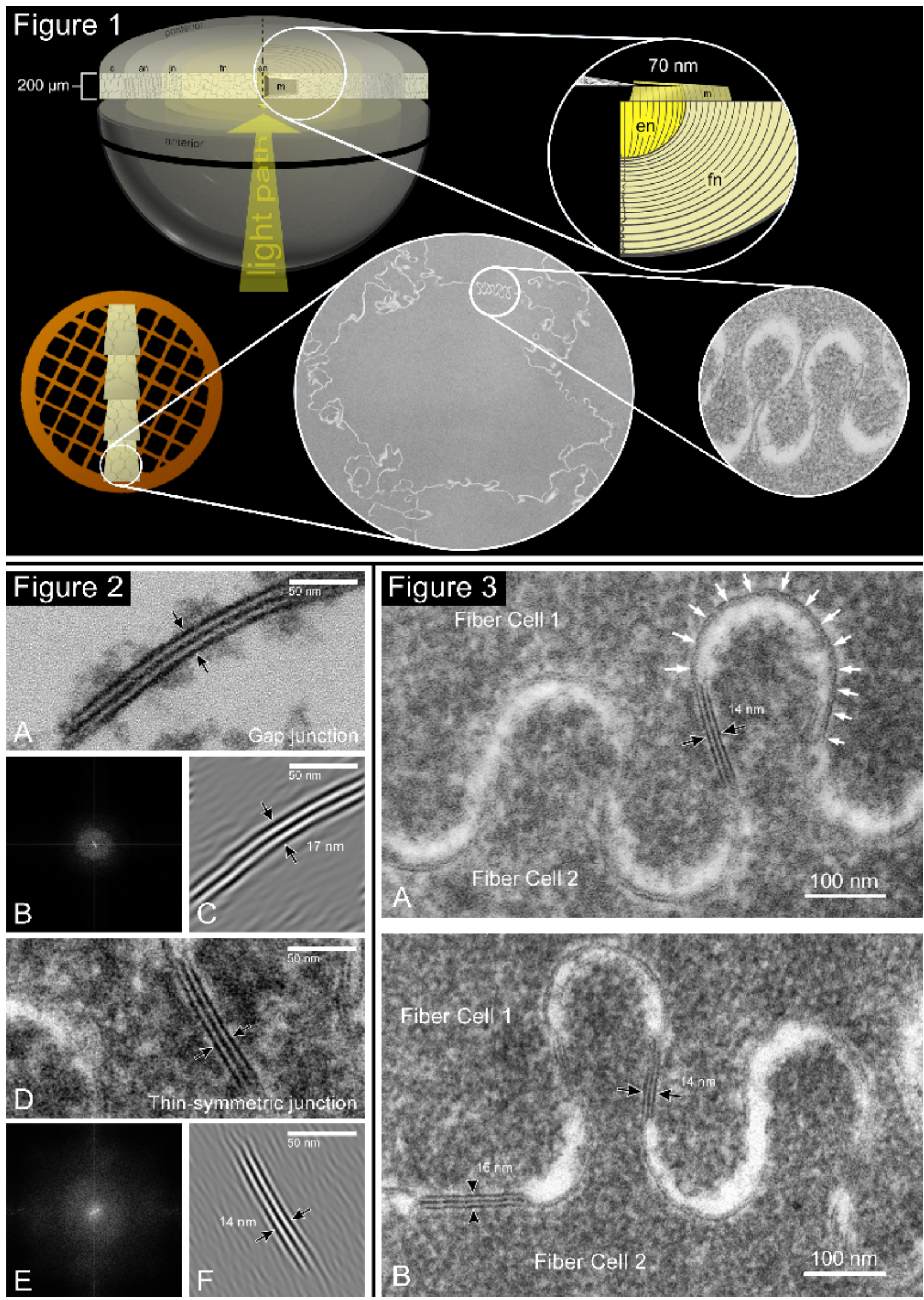

\section{Figure 3}
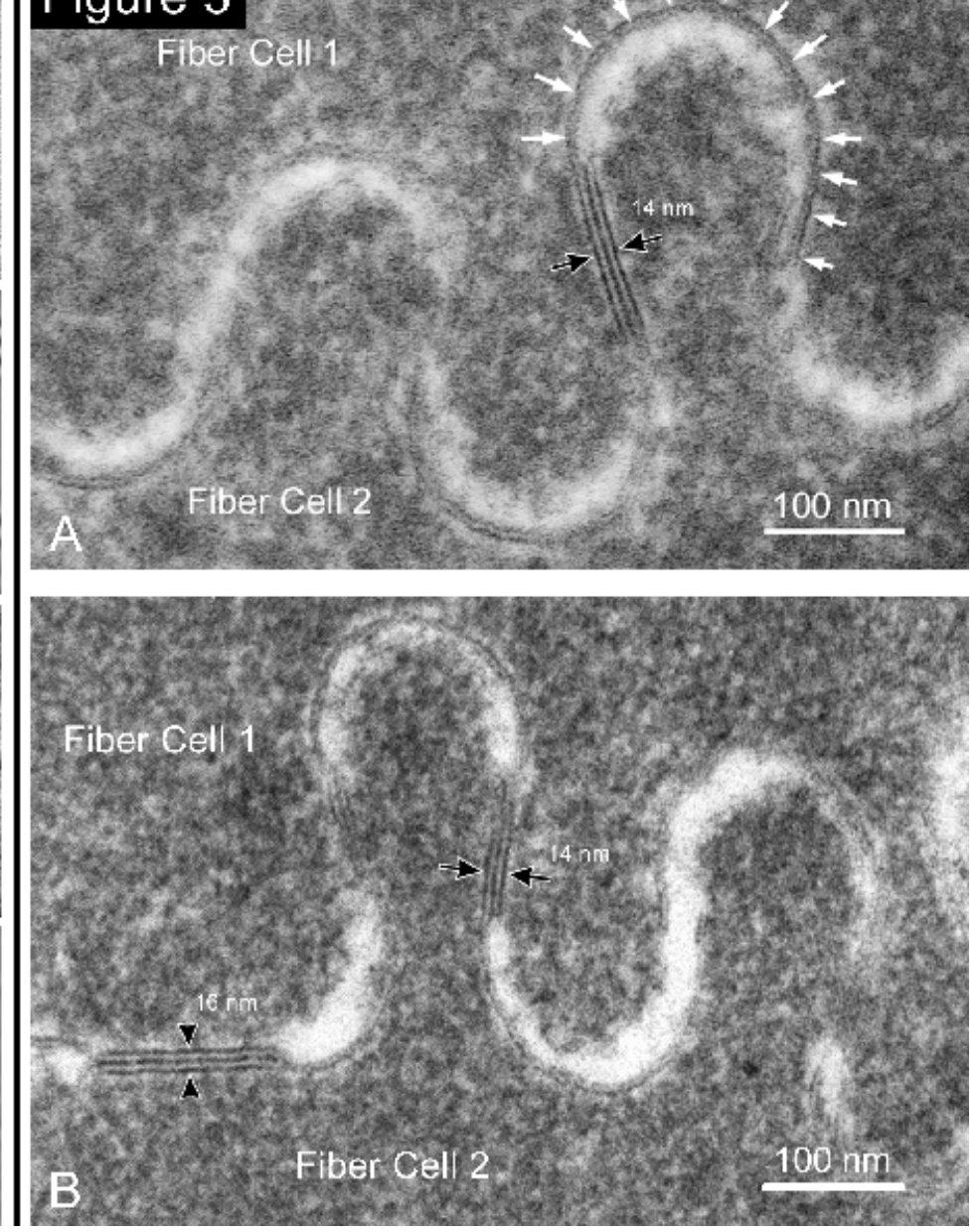